\title{
1 A prototype sensor for the assessment of soil bulk density
}

2 Mohammed Z. Quraishi, Abdul M. Mouazen*

3 Environmental Science and Technology Department, Cranfield University, Bedfordshire, MK43 0AL, United

$4 \quad$ Kingdom

5

6 Abstract

7 A prototype bulk density sensor (PBDS) to assess soil bulk density (BD) has been developed 8 and tested for top soil $(0-15 \mathrm{~cm})$. It is a multi-sensor kit, consisting of a penetrometer 9 equipped with a visible and near-infrared (vis-NIR) spectrophotometer. Artificial neural network (ANN) was used to develop a BD prediction model, as a function of penetration

11 resistance (PR), soil moisture content (MC), organic matter content (OMC) and clay content 12 (CLC), using 471 samples collected from various fields across four European countries, 13 namely, Czech Republic, Denmark, the Netherlands and the UK. While penetration resistance 14 (PR) was measured with a standard penetrometer (30 degree cone of $1.26 \mathrm{~cm}^{2}$ cone-base area), MC, OMC and CLC were predicted with a vis-NIR $(1650-2500 \mathrm{~nm})$ spectrophotometer (Avantes, Eerbeek, the Netherlands). ANN was also used to model the vis-NIR spectra to predict MC, OMC and CLC. The PBDS was validated by predicting topsoil $(0-0.15 \mathrm{~m}) \mathrm{BD}$ of three selected validation fields in Silsoe experimental farm.

19 The ANN BD model performed very well in training (coefficient of determination $\left(\mathrm{R}^{2}\right)=$ 0.92 and root mean square error $\left.(\mathrm{RMSE})=0.05 \mathrm{Mg} \mathrm{m}^{-3}\right)$, validation $\left(\mathrm{R}^{2}=0.84\right.$ and $\mathrm{RMSE}=$

$\left.210.08 \mathrm{Mg} \mathrm{m}^{-3}\right)$ and testing $\left(\mathrm{R}^{2}=0.94\right.$ and $\left.\mathrm{RMSE}=0.04 \mathrm{Mg} \mathrm{m}^{-3}\right)$. The validation of PBDS for 22 BD assessment in the three validation fields provided high prediction accuracy, with the 23 highest accuracy obtained in Downing field $\left(\mathrm{R}^{2}=0.95\right.$ and $\left.\mathrm{RMSE}=0.02 \mathrm{Mg} \mathrm{m}^{-3}\right)$. It can be concluded that the new prototype sensor to predict BD based on, a standard penetrometer 
equipped with a vis-NIR spectrophotometer and ANN model can be used for in situ assessment of BD. The PBDS can also be recommended to provide information about soil MC, OMC and CLC, as the ANN vis-NIR calibration models of these properties were of excellent performance.

*Corresponding author. Tel.: +44 1234750111 fax: +44 1234752971.

E-mail address: a.mouazen@cranfield.ac.uk (A. M. Mouazen).

Keywords: bulk density, vis-NIR spectroscopy, in situ measurement, penetrometer, artificial neural network.

\section{Introduction}

Soil strength is a dynamic property that changes with time and space under the influences of climate, soil management practices and plant growth (Koolen and Kuipers, 1983). Soil deformation following a single or multiple passes of heavy agriculture machinery results in soil compaction and structure deterioration, which leads to increase in soil strength, reduction in hydraulic conductivity and infiltration rate, and poor root penetration and plant growth (Franzen et al., 1994; Quraishi and Mouazen, 2013a). Random traffic of heavy machinery during harvest also causes long lasting damage to the soil structure because of deep penetration of downward forces causing deep compaction (Ekwue and Stone, 1995). Deep compaction is difficult to ameliorate, since natural and biological activities are limited at deep soil horizons. Subsoiling is also of limited impact particularly if carried out under heavy and wet soil conditions. Due to the dynamic nature of the soil, soil strength is affected by soil moisture content (MC), organic matter content (OMC), degree of compaction and texture to name a few. 
One of the properties to characterise soil compaction is BD (Mouazen and Ramon, 2002), which does not necessarily reflect soil function. Core sampling of a known volume of soil is utilised for the measurement of soil BD (British Standards, 2011), based on drying of the soil cylinder at $105{ }^{\circ} \mathrm{C}$ for $24 \mathrm{~h}$. The disadvantageous of this method are that it is very difficult, labour intensive, time costly procedure and prone to measurement error, particularly under dry soil conditions (Mouazen and Ramon, 2006; Quraishi and Mouazen, 2013a). An innovative approach to assess $\mathrm{BD}$ based on a complex interrelationship between $\mathrm{BD}, \mathrm{MC}$, OMC, clay content (CLC) and penetration resistance (PR) was recently introduced by Quraishi and Mouazen (2013b). They used artificial neural network (ANN) to develop a model to assess $\mathrm{BD}$ as a function of PR, MC, OMC and CLC. This model enabled the assessment of BD based on traditional laboratory methods of soil analyses in addition to field measured PR (coefficient of determination $\left(\mathrm{R}^{2}\right)$ of 0.81 and root mean square error (RMSE) of $0.11 \mathrm{Mg} \mathrm{m}^{-3}$ ). However, since soil samples had to be collected in the field where PR is measured, and transferred to the laboratory for the traditional analyses of OMC, MC and CLC, it was concluded that this method did not overcome the disadvantages of the core sampling method of being expensive, slow and labour intensive. Therefore, Quraishi and Mouazen (2013c) has replaced the traditionally measurement methods of MC, OMC and CLC with visible and near infrared (vis-NIR) spectroscopy. By substituting vis-NIR predicted values of MC, OMC and CLC into ANN BD prediction model, authors reported successful prediction of topsoil $\mathrm{BD}\left(\mathrm{R}^{2}\right.$ of 0.80 and RMSE of $\left.0.08 \mathrm{Mg} \mathrm{m}^{-3}\right)$. They confirmed that the proposed methodology is capable of overcoming the disadvantages of the traditional core sampling method of BD measurement, as vis-NIR spectroscopy enables cost effective and fast prediction of soil properties (Mouazen et al., 2005, 2007, 2009). At this stage, this new methodology requires the development of an instrumentation to enable in situ acquisition of multiple georeferenced data, including PR and vis-NIR spectra, to be fed as input data into 
models to predict $\mathrm{BD}$, as a function of measured PR and vis-NIR predicted MC, OMC and CLC.

The aim of this paper was to design and validate a prototype BD sensor (PBDS), as a new tool for rapid, cost effective and in situ assessment of BD, as a function of measured PR, and vis-NIR predicted MC, OMC and CLC.

\section{Materials and methods}

\subsection{Field measurement and soil sampling}

Field measurement of topsoil $(0-15 \mathrm{~cm}$ depth) PR and BD was carried out in summer of 2010, 2011 and 2012, in 19 fields across different Europe countries as shown in Table 1 (Quraishi and Mouazen, 2013a, 2013b \& 2013c). Avenue, Orchard, Ivy ground, Beechwood, Clover hill, Upbury, Chipping and Downing fields are situated at Silsoe experimental farm, Cranfield University, the UK. Two fields were part of a Research Station for arable farming and field production of vegetables in Lelystad, the Netherlands. Two other fields were located at Wageningen University, Wageningen, the Netherlands. One field in Czech Republic and two fields in Denmark were measured in 2010 as part of FutureFarm FP7 project (http://www.futurefarm.eu/). Measurement at Odstone field in Leicestershire, the UK was carried out in a grassland field. Three fields were measured at Duckend Farm near Bedford in Bedfordshire, the UK. Figure 1 and Table 1 show the texture classes of all fields used in this study.

Soil BD was measured using Kopecki ring core sampling kit, whereas PR measurement was carried out with Eijkelkamp penetrologger with a 30 degree cone of $1.26 \mathrm{~cm}^{2}$ cone-base area (Eijkelkamp, 2009) in 2010 and 2011. In 2012, PR was measured using a new prototype penetrometer designed in this study, which is explained below. The number of samples collected from each field varied, depending on the size of the field, but ranged from 4 to 48 
99 (Table 1). At each sampling point, three PR measurements, one bulk soil sample and one BD 100 core sample were collected. The PR measurement was carried out within half a meter

101 distance from the BD core sample location, ensuring that both measurements were taken

102 either in or outside a wheel rut. The PR readings were averaged in one reading (Quraishi and

103 Mouazen, 2013b). A total of 408 bulk soil samples and BD core samples were collected in

1042010 and 2011. These samples were used to develop a general calibration model to predict 105 BD. Three additional field measurements were carried out in 2012 to validate the 106 measurement accuracy of PBDS using the general calibration model. These fields were Ivy

107 Ground, Chipping and Downing (Table 1), all in Silsoe experimental farm. In total, 87 108 samples were collected from these three fields using the PBDS. Out of the 87 locations, BD 109 was measured at 63 sampling points only using a Kopecki ring kit.

110

\section{$111 \quad 2.2$ Prototype bulk density sensor (PBDS)}

112 The PBDS was designed and developed to predict multiple soil properties in addition to BD.

113 It consists of a rod and cone assembly connected to a load cell, which has a maximum load of

1141000 N. A 50 channel global positioning system (GPS) was used to record the sampling

115 location. The 30 degree, $1.26 \mathrm{~cm}^{2}$ base-area cone connected to the rod were assembled with a

116 fibre type standalone vis-NIR spectrophotometer $(1650-2500 \mathrm{~nm})$ (Avantes, Eerbeek, The

117 Netherlands), as shown in Fig. 2. Light illumination fibre was connected to a 10 watt halogen 118 lamp, whereas detection fibres were connected to 256 pixel Indium Gallium Arsenide 119 (InGaAs) detector. The resultant spectra were of $7 \mathrm{~nm}$ resolution, and consisted of 256 120 wavelengths. The selection of $1650-2500 \mathrm{~nm}$ wave range spectrophotometer was based on 121 previous studies confirming this range to be the most significant for MC, OMC and CLC 122 prediction with vis-NIR spectroscopy (Stenberg et al., 2010; Kuang et al., 2012). This multisensor instrumentation was connected to a laptop for data logging using AvaSoft 7.7 software 
124 (Avantes, Eerbeek, the Netherlands) (Fig. 2). The optical assembly of the PBDS was first tested in the laboratory under controlled conditions by inserting the cone in the soil placed in black containers to avoid the effect of ambient light. After successful laboratory testing, the sensor was tested and validated in the three validation fields in Silsoe experimental farm in 2012 (Table 1). The PBDS was inserted in the soil at a constant speed to a depth of $20 \mathrm{~cm}$, along which the vis-NIR soil spectra and PR were recorded at a sampling resolution of $10 \mathrm{~Hz}$.

\subsection{Laboratory analyses}

132 Soil samples collected from all fields (Table 1) were analysed for MC, OMC and average 133 field CLC using oven drying (British Standards, 2007), loss of ignition (British Standards, 134 2000), and particle size distribution (British Standards, 1998) methods, respectively. Soil BD 135 were measured by the oven drying method (British Standards, 2007), by drying the samples at $105{ }^{\circ} \mathrm{C}$ for $24 \mathrm{~h}$ to obtain MC and calculate dry BD (British Standards, 2011).

\subsection{Establishment of visible and near infrared (vis-NIR) calibration} models

140 Two separate sample sets collected from the UK were used for vis-NIR spectra modelling.

141 The first set was used to develop calibration models of MC and OMC, whereas the second set

142 was used to develop CLC model. Samples for the first set were collected from Beechwood,

143 Clover Hill, Upbury, Ivy Ground, Chipping and Downing fields in Cranfield experimental

144 farm in Silsoe (Table 2). In total, 111 samples were collected from these six fields to form a

145 farm-scale (Silsoe farm) calibration models for MC and OMC. The second set consisting of

14663 samples (Aldhumayri, 2012; Alhwaimel, 2013) were collected from Silsoe farm (e.g.

147 Avenue, Orchard, Ivy ground, Chipping and Downing fields), a farm in Lincolnshire 
148 (Vicarage, Marshalls, and Thetford fields), a farm in Cambridgeshire (Ely and Wypemere

149 fields), a field in Norfolk (Elveden field) and a field in Shropshire (Shropshire). Therefore,

150 samples used to develop the CLC model can be classified as multi-county-scale model.

151 All samples listed in Table 2 were scanned in the laboratory with the same fibre type vis-NIR

152 spectrophotometer of PBDS (Avantes, Eerbeek, The Netherlands), linked with the

153 penetrometer cone (Fig. 2). Before scanning, stones and plant residues were removed from

154 the soil samples and placed in a glass container after mixing. This was done to exclude

155 interference of stone and plant debris in soil spectra. Each sample was scanned 10 times in

156 diffuse reflectance mode. White reference was used before scanning and at 30 min interval to

157 re-calibrate the spectrophotometer.

2.4.1 Spectra pre-processing and development of visible and near infrared (vis-NIR) calibration models

161 After averaging the 10 spectra of each soil sample in one spectrum, the resultant spectra were smoothed by averaging 5 successive wavelengths. The spectra were then exported to

163 Microsoft Excel 2010, where the noise from either end of the spectra was removed and 164 remaining range of 1653-2498 nm was withheld. STATISTICA 11 ANN toolbox (StatSoft, 165 Inc., Tulsa, USA) was used to establish calibration models for MC, OMC and CLC, using spectra of the samples listed in Table 2. The networks were multilayer perceptron (MLP)

167 ANN and used Broyden-Fletcher-Goldfarb-Shanno (BFGS) training algorithm with very fast 168 convergence (StatSoft, 2011). The hidden and output layers consisted of hyperbolic tangent 169 (Tanh) transfer function, since it produced the best results, compared to other transfer 170 functions.

171 ANN models for MC, OMC and CLC were developed using 60, 20 and 20\% of the first (e.g.

172111 samples for MC and OMC) and second (e.g. 63 samples for CLC) sample sets (Table 2) 
173 in training, validation and testing, respectively. The validation of ANN models consisted of

174 re-aligning the weights and biases of the training model, whereas the testing phase was to

175 simply test the network with the test dataset. A total of 100 ANN networks were trained, out 176 of which 5 networks were selected for further analysis. The prediction performances of these

177 models were evaluated by means of $R^{2}$ and RMSE. Residual prediction deviation (RPD),

178 which is the ratio of standard deviation of reference measured values (for training, validation 179 or test sets) divided by the RMSE was used to compare between different calibration models

180 developed (Williams, 1987, Stenberg et al., 2004, Viscarra Rossel et al., 2006). Table 3 181 shows the classification adopted for this study based on RPD values as stated by Viscarra 182 Rossel et al. (2006).

183

\subsection{Development of bulk density model}

185 A model to predict BD (dependent variable), as a function of PR, MC, OMC and CLC 186 (independent variables) was developed with ANN, using STATISTICA 11 ANN toolbox 187 (StatSoft, Inc., Tulsa, USA). The network was a MLP ANN using the BFGS training 188 algorithm. A hyperbolic tangent (Tanh) was utilised as the hidden and output activation 189 function, since it produced the best results compared to other activation functions, such as exponential and logarithmic functions (Quraishi and Mouazen, 2013b).

191 The values of MC, OMC and CLC used as input for ANN were obtained from laboratory 192 reference measurement for all fields measured in 2010 and 2011 (Table 1), apart from

193 Duckend 1-3 (Gonzales et al., 2013), Clover Hill, Beechwood, Upbury, Ivy Ground (2011), 194 whose MC, OMC and CLC were predicted using vis-NIR calibration models. These models were also used for data from Chipping, Ivy Ground and Downing fields collected in 2012. In total, 471 samples (408 samples from 2010 and 2011, and 63 samples from 2012) were used

197 to develop and validate the BD model. These samples were randomly divided into training 
198 (60\%), validation (20\%) and test (20\%) sets. The prediction performance of the BD model was evaluated by means of $R^{2}$ and RMSE.

200

201

\subsection{Field mapping}

Maps of BD, MC and OMC were developed for Downing field only, as an example. In this field 48 points were measured with the PBDS, out of which 24 points were selected to collect core samples with the Kopecki ring kit. Two types of maps were developed for each property, namely, full-point maps and comparison maps. The former maps were based on 48 points of BD, MC and OMC measured with the PBDS (Fig. 3), whereas the latter maps were based on 24 points collected either with Kopecki ring or PBDS (Fig. 3).

Semi-variograms analysis was carried out for the comparison and full-point maps using

Vesper 1.63 software developed by the Australian Centre for Precision Agriculture (Minasny et al., 2005). Spherical model (Eqn. 1) was adopted to calculate semi-variance, since it resulted in the lowest root mean square error of prediction (RMSEP).

212

$$
\gamma=C_{0}+\left(C_{1} \times\left(\frac{3 h}{2 A}-\frac{h^{3}}{2 A^{3}}\right)\right) \quad 0<h<A
$$

214 Where, $\gamma$ is semi-variance, $C_{0}$ is the nugget value, $C_{l}$ is sill, $h$ is the lag distance, and $A$ is 215 range.

216 Based on the variogram data, maps of BD, MC and OMC were developed with ArcGIS 217 ArcMap (ESRI ArcGIS ${ }^{\mathrm{TM}}$ version 10, CA, USA). Ordinary kriging with the semi-variogram 218 data (Table 4) was performed to map the spatial variation. Minimum and maximum 219 neighbours during the analyses were set to two and five, respectively. For the comparison 220 maps, variogram model of the reference measured BD, MC and OMC was used to perform 
ordinary kriging. Predicted BD, MC and OMC maps were later developed using the same models. Error map of BD was also developed by subtracting measured and predicted values of the 24 validation points. For the full-point maps, predicted values of $\mathrm{BD}, \mathrm{MC}$ and $\mathrm{OMC}$ were used for ordinary kriging based on semi-variogram data listed in Table 4 . On the basis of average nearest distance, a lag size of $4.92 \mathrm{~m}$ was selected for all three properties. The range was adjusted to reduce the RMSEP for the spatial prediction with a grid size of $1 \mathrm{~m}$.

\section{Results and discussion}

\subsection{Performance of visible and near infrared (vis-NIR) general calibration} models

Table 5 shows the prediction accuracy of MC, OMC and CLC general calibration models for the training, validation and test sets. Figure 4 shows scatter plot of measured against predicted values of $\mathrm{MC}, \mathrm{OMC}$ and $\mathrm{CLC}$ for the test set. The results reveal that the performance of all vis-NIR general calibration models of the three soil properties is classified as excellent (RPD > 2.5), except for MC in the test set, where the performance is found to be very good $(\mathrm{RPD}=2.46)$.

\subsubsection{Moisture content model}

239 Values of RMSE, RPD and $\mathrm{R}^{2}$ for the prediction of MC in the test set are $2.60 \%, 4.03$ and 240 0.94, respectively, which are of similar magnitude to those obtained by Mouazen et al. (2006)

241 for multiple-field scale calibration $\left(\mathrm{RMSE}=2.10 \%, \mathrm{RPD}=3.22\right.$ and $\left.\mathrm{R}^{2}=0.91\right)$. Although a smaller wavelength range was used in the current study $(1650-2500 \mathrm{~nm})$, as compared to

243 that used by Mouazen et al. (2006) $(350-2500 \mathrm{~nm})$, the ANN adopted in the current study 
244 improves the prediction accuracy of vis-NIR spectroscopy, as compared to partial least 245 squares (PLS) regression analysis adopted by Mouazen et al. (2006), which was also 246 confirmed by Mouazen et al. (2010). Upadhyaya et al. (1994), Slaughter et al. (2001) and

247 Ben-Dor et al. (2008) also reported similar PLS model accuracy for MC prediction with $\mathrm{R}^{2}$ 248 values of $0.99,0.97$ and 0.98 , respectively, for independent validation sets.

\subsubsection{Organic matter content model}

The OMC is predicted with a lower accuracy $(\mathrm{RMSE}=0.82 \%)$ in comparison to MC (Table in clay fields is considerably higher $(8.03 \%)$ than that in sandy loam fields $(3.26 \%)$, which results in empty gap in OMC range (Fig. 4). Although a high $\mathrm{R}^{2}$ value of 0.96 is calculated, a large slop and intercept can be observed. However, the small RMSE value of $0.82 \%$ confirms the model applicability to predict OMC, especially with RPD value of 2.46 , which is classified as a very good quantitative model for prediction (Table 3). Ben-Dor et al. (2008) reported a $\mathrm{R}^{2}$ of 0.94 and a RMSE of $8.7 \%$ for independent validation, which is less accurate than the results achieved in current study $\left(\mathrm{R}^{2}=0.96\right.$ and $\left.\mathrm{RMSE}=0.82 \%\right)$.

\subsubsection{Clay content model}

262 The performance of the clay model for the prediction of CLC for the test set is encouraging $\left(\mathrm{R}^{2}=0.92 ; \mathrm{RMSE}=4.53 \%\right.$ and $\left.\mathrm{RPD}=3.68\right)$. Waiser and Morgan (2007) reported in situ

264 prediction of CLC for field moist soils with smaller accuracy $\left(\mathrm{R}^{2}=0.83, \mathrm{RMSE}=6.1 \%\right.$ and 265 RPD = 2.36). Bricklemyer and Brown (2010) also predicted clay using lab measured spectra 266 but also with reduced accuracy $\left(\mathrm{R}^{2}=0.75, \mathrm{RMSE}=5.16 \%\right.$, and $\left.\mathrm{RPD}=1.8\right)$, as compared to those obtained in the current study (Table 5). Both authors used PLS regression, whereas 
ANN was adopted in the current study, which proves that ANN is a more robust and provide more accurate estimations when compared to PLS regression (Mouazen et al., 2010).

270

271

\subsection{Performance of general bulk density (BD) model}

272 The general ANN BD model performs notably well in training, validation and testing (Fig. 5

$273 \&$ Table 6), with excellent performance in testing $\left(\mathrm{R}^{2}=0.94, \mathrm{RMSE}=0.04 \mathrm{Mg} \mathrm{m}^{-3}\right)$. The

274 high vis-NIR prediction accuracies of MC, OMC and CLC reported in this study reinforces 275 the high accuracy achieved for the prediction of BD. Quraishi and Mouazen (2013b) stated that the sum of error attributed to the laboratory reference analyses of MC, OMC and CLC accounted for $9 \%$ of the total error. It is suggested that by combining the vis-NIR spectroscopy coupled with ANN to predict MC, OMC and CLC, the error associated with laboratory analysis is avoided. This might explain the decrease in RMSE of BD prediction from $0.11 \mathrm{Mg} \mathrm{m}^{-3}$ (Quraishi and Mouazen, 2013b) to $0.04 \mathrm{Mg} \mathrm{m}^{-3}$ (Table 6), for input data about soil properties obtained from laboratory analysis and vis-NIR spectroscopy, respectively.

\subsection{Validation of prototype bulk density sensor (PBDS)}

The BD values of 63 samples collected from Ivy ground (2012), Chipping, and Downing fields were predicted using the BD model developed with ANN, based on the input data of PR measured with the PBDS, and vis-NIR predicted values of MC OMC and CLC. The visNIR spectroscopy shows high prediction accuracy for MC, OMC and CLC for all three validation fields, when compared with the standard laboratory measurement methods using samples collected from the same sampling positions (Table 7). For the CLC model, the error calculated for all three fields is less than $8 \%$ of the average field CLC value. Since the soil 
sample used for CLC model was an average of the whole field, it was not possible to compare point-by-point predicted with measured CLC. The scatter plots in Fig. 6 shows a good fit between the measured and predicted values of MC and OMC for each validation field. Best results are obtained for Chipping field with the lowest RMSE for both MC (0.32\%) and OMC $(0.09 \%)$. The measurement accuracy of the clayey soil Ivy field is similar to the other two fields (RMSE $=0.51 \%$ and $0.11 \%$, respectively). In Downing field, the prediction accuracy for both $\mathrm{MC}(\mathrm{RMSE}=0.60 \%$; $\mathrm{RPD}=3.68)$ and $\mathrm{OMC}(\mathrm{RMSE}=0.12 \%$; $\mathrm{RPD}=2.74)$ is excellent, but less accurate than the other two fields (Table 7).

In addition to the possibility of measuring MC, OMC and CLC with the PBDS, BD can also

301 be assessed. The predicted and measured BD in Downing field are in a good agreement $\left(\mathrm{R}^{2}=\right.$

3020.95 and $\mathrm{RMSE}=0.02 \mathrm{Mg} \mathrm{m}^{-3}$ ). Less satisfactorily prediction performances are observed in

303 Ivy and Chipping fields with RMSE of 0.04 and $0.03 \mathrm{Mg} \mathrm{m}^{-3}$, respectively (Table 6 and Fig.

304 7). Literature shows no similar studies about the assessment of BD, as a function of PR, MC, OMC and CLC measured with a PBDS. Therefore, the PBDS system introduced in the current study proves to be unique in the assessment of BD as well as the prediction of other properties that are relevant for land management.

\subsection{Field mapping}

\subsubsection{Comparison maps}

311 Comparison maps of measured and predicted BD, MC and OMC were developed for

312 Downing field only, as an example. The semivariograms parameters for studied soil

313 properties are shown in Fig. 8 and Table 4. The kriging method used was similar to that of

314 Quraishi and Mouazen (2013a), but spherical semivariance model was used in the current 315 work instead of exponential semivariance model. Mouazen and Ramon (2006) also carried 
316 out similar investigation to compare measured and predicted maps of soil properties for sandy

317 loam soil. An equal maximum lag distance of $41 \mathrm{~m}$ is calculated for BD and MC, whereas a

318 maximum lag distance of $65 \mathrm{~m}$ is calculated for OMC, which is attributed to low variation of

319 OMC throughout the field.

320 Figure 9 compares between the spatial distributions of measured and predicted BD. It can be

321 observed that BD varies throughout the field, with high BD values encountered in the top left, top and bottom right corner of the field. The predicted BD map shows similar spatial patterns to the corresponding measured map, with a similar range of 1.40 to $1.67 \mathrm{Mg} \mathrm{m}^{-3}$. The error map shown in Fig. 9c illustrates that the maximum error is encountered in the top left, top and bottom right hand side of the field. The error ranges from -0.054 to $-0.001 \mathrm{Mg} \mathrm{m}^{-3}$.

The measured and predicted MC maps (Figs. 10a and 10b, respectively) show very similar spatial patterns, which can be attributed to the high match between vis-NIR predicted and measured MC (Fig. 6 \& Table 7). It can be observed that MC gradually decreases from the top right corner to the bottom left corner. The error map in Fig. 10c illustrates that the error ranges between -0.86 to $1.76 \%$, with the largest negative and positive errors encountered towards the central area, and left and right hand side areas of the field, respectively. The comparison maps between the measured and predicted OMC (Fig. 11a and 11b, respectively) also illustrate similar spatial variation pattern, which is also similar to MC distribution pattern. A large area of high OMC can be observed at the right hand side of the field. The low band of OMC towards the left hand side of the field is identical to that of MC. Indeed, OMC diminishes from top right corner to bottom left corner of the field, which is inline with that of the MC variation. The error map in Fig. 11c also shows negative error at the central area (-0.18 to $-0.05 \%)$, whereas positive error can be observed at the right and left hand side $(0.05$ to $0.19 \%)$ of the field. The spatial pattern of OMC map is similar to that of 
340 MC, which can be explained by the positive correlation between the two properties (Quraishi and Mouazen, 2013b).

\subsubsection{Full-point maps}

344 The full-point maps for BD, MC and OMC were generated using 48 points predicted with the

345 new BDPS. The spherical semivariograms used for kriging are shown in Fig 12, whose

346 properties are listed in Table 4. Full-point maps (Fig. 13) show more detailed information in 347 comparison to the corresponding comparison maps (Figs. 9, 10 and 11) due to the higher resolution of sampling points of the former maps (Mouazen and Ramon, 2009; Quraishi and Mouazen, 2013a). It can be observed that the majority of the field BD lies between 1.36 to $1.60 \mathrm{Mg} \mathrm{m}^{-3}$ (about $80 \%$ of the field area). This part of the field requires minimal tillage operations according to the packing density criteria, which states that soils with a packing density of $1.40 \mathrm{Mg} \mathrm{m}^{-3}$ (equivalent to a BD of $1.36 \mathrm{Mg} \mathrm{m}^{-3}$ for $10 \% \mathrm{CLC}$ ) to $1.75 \mathrm{Mg} \mathrm{m}^{-3}$ (equivalent to a $\mathrm{BD}$ of $1.60 \mathrm{Mg} \mathrm{m}^{-3}$ for $10 \% \mathrm{CLC}$ ) is neither strong nor loose soil (Hodgson, 1974). A small part of the field with a larger BD than $1.60 \mathrm{Mg} \mathrm{m}^{-3}$ (about $20 \%$ of the field area) will require more aggressive tillage intervention. Both BD and MC maps illustrates high values in the upper side of the field, where heavy traffic and surface water flow from the adjacent road take place. Avoiding or reducing damage to the soil at this part will preserve good soil structure for plant growth and water infiltration. The OMC map provides less obvious spatial similarity to MC map than corresponding comparison maps produced with a

360 smaller number of points of 24 . This necessitates the need to increase the sampling 361 resolutions to allow for a better understanding of the spatial variation in soil properties. The sampling resolution will depend on the size of the field, cost involved for sample analysis, and other requirements associated with the land management practices. With the PBDS 
proposed in this study, the collection of a large number of sampling points for the analysis of multi-soil properties, quickly and in a cost-effective manner becomes possible.

366

367

\section{Conclusions}

A new prototype bulk density sensor (PBDS) to predict bulk density (BD), as a function of in situ measured PR and visible and near infrared (vis-NIR) predicted moisture content (MC), organic matter content (OMC) and clay content (CLC) was developed and tested in three fields in the UK. Artificial neural network (ANN) was implemented to establish BD model, as a function of vis-NIR predicted MC, OMC and CLC. Results allowed the following conclusions to be drawn:

1. The vis-NIR general calibration models of MC, OMC and CLC provided excellent quantitative prediction accuracies with ratio of prediction deviation (RPD) of 5.86, 7.84 and 4.94 , respectively.

2. The independent testing of MC and OMC models performance in the validation fields demonstrated high accuracy for MC $\left(\mathrm{R}^{2}=0.94\right.$ and $\left.\mathrm{RMSE}=0.32 \%\right)$ and $\mathrm{OMC}\left(\mathrm{R}^{2}=\right.$ 0.90 and $\mathrm{RMSE}=0.09 \%$ ).

3. The performance of the BD general calibration model was found to be promising, with $\mathrm{R}^{2}$ of 0.94 and RMSE of $0.04 \mathrm{Mg} \mathrm{m}^{-3}$ in the test set.

4. Predicted BD with the new PBDS showed very good correlation with measured values $\left(\mathrm{R}^{2}=0.95\right.$ and $\left.\mathrm{RMSE}=0.02 \mathrm{Mg} \mathrm{m}^{-3}\right)$.

5. Comparison maps between measured and PBDS predicted soil properties showed high spatial similarities. The full-point maps based on double number of points of 48 provided more detailed information than the comparison maps (24 points). The fast 
and cost effective sampling provided by the PBDS introduced in this study will support a high resolution mapping of the spatial variation in soil properties.

389 The new PBDS requires further validation in new fields. Furthermore, the BD model will be

390 updated for new soil texture classes such as silt, silty clay and sandy clay to broaden the

391 applicability of the approach. This system can be then further developed for evaluations

392 throughout the soil profile.

393

394 Acknowledgments

395 Authors acknowledge the financial support of the UK Engineering and Physical Science 396 Research Council (EPSRC) and The Douglas Bomford Trust.

397 


\section{References}

1. Aldhumayri, M.H., 2012. Optimizing position of moisture sensors by mapping of clay, moisture content and organic carbon content. MSc by Research Thesis, Cranfield University, UK.

2. Alhwaimel, S.A., 2013. Fusion of multi soil data for the delineation of management zones for variable rate irrigation. MSc by Research Thesis, Cranfield University, UK.

3. Ben-Dor, E., Heller, D., Chudnovsky, A., 2008. A novel method of classifying soil profiles in the field using optical means, Soil Science Society of America Journal, 72, 1113-1123.

4. Bricklemyer, R.S., Brown, D.J., 2010. On-the-go VisNIR: Potential and limitations for mapping soil clay and organic carbon, Computer and Electronics in Agriculture,

5. British Standards, 2011. Soil improvers and growing media: BS EN 13041:2011. Determination of physical properties. Dry bulk density, air volume, water volume, shrinkage value and total pore space. British Standards Institution, UK.

6. British Standards, 2007. Soil improvers and growing media: BS EN 13040:2007. Sample preparation for chemical and physical tests, determination of dry matter content, moisture content and laboratory compacted bulk density. British Standards Institution, UK.

7. British Standards, 2000. Soil improvers and growing media: BS EN 13039:2000. Determination of organic matter content and ash. British Standards Institution, UK. methods. Section 5.4: Determination of particle size distribution in mineral soil material - method by sieving and sedimentation. British Standards Institution, UK.

9. Eijkelkamp, 2009. P1.52-1 Penetrologger. 2009. 
10. Ekwue, E.I., Stone, R.J., 1995. Organic matter effects on the strength properties of compacted agricultural soils, Transactions - American Society of Agricultural Engineers, 38, 357-365.

11. Franzen, H., Lal, R., Ehlers, W., 1994. Tillage and mulching effects on physical properties of a tropical Alfisol. Soil \& Tillage Research 28, 329-346.

12. González, O.M., Kuang, B., Quraishi, M.Z., García , M.Á.M., Mouazen, A.M., 2013. On-line measurement of soil properties without direct spectral response in near infrared spectral range. Soil \& Tillage Research, 132, 21-29.

13. Hodgson, J.M., 1974. Soil survey field handbook: describing and sampling soil profiles, Rothamsted experimental station, Lawes agricultural trust, Harpenden.

14. Koolen, A.J., Kuipers, H., 1983. Agricultural Soil Mechanics. Springer, Berlin, Germany.

15. Kuang, B., Mahmood, H.S., Quraishi, M.Z., Hoogmoed, W.B., Mouazen, A.M., van Henten, E.J., 2012. Chapter four - Sensing Soil Properties in the Laboratory, In Situ, and On-Line: A Review, in: Advances in Agronomy. Academic Press, UK, 155-223.

16. Minasny, B., McBratney, A.B., Whelan, B.M., 2005. Vesper 1.62 - Spatial Prediction Software for Precision Agriculture.

17. Mouazen, A.M., Ramon, H., 2002. A numerical-statistical hybrid modelling scheme for evaluation of draught requirements of a subsoiler cutting a sandy loam soil, as affected by moisture content, bulk density and depth. Soil \& Tillage Research, 63, $155-165$.

18. Mouazen, A.M., De Baerdemaeker, J., Ramon, H., 2005. Towards development of on446 line soil moisture content sensor using a fibre-type NIR spectrophotometer, Soil and Tillage Research, 80, 171-183. 
19. Mouazen, A.M., Ramon, H., 2006. Development of on-line measurement system of bulk density based on on-line measured draught, depth and soil moisture content. Soil Tillage Res. 86, 218-229.

20. Mouazen, A.M., Maleki, M.R., De Baerdemaeker, J., Ramon, H., 2006. On-line measurement of some selected soil properties using a Vis-NIR sensor. Soil \& Tillage Research, 93, 13-27.

21. Mouazen, A.M., Maleki, M.R., De Baerdemaeker, J., Ramon, H., 2007. On-line measurement of some selected soil properties using a VIS-NIR sensor, Soil and Tillage Research, 93, 13-27.

22. Mouazen, A.M., Maleki, M.R., Cockx, L., Van Meirvenne, M., Van Holm, L.H.J., Merckx, R., Ramon, H., 2009. Optimum three-point linkage set up for improving the quality of soil spectra and the accuracy of soil phosphorus measured using an on-line visible and near infrared sensor, Soil and Tillage Research, 103, 144-152.

23. Mouazen, A.M., Ramon, H., 2009. Expanding implementation of an on-line measurement system of topsoil compaction in loamy sand, loam, silt loam and silt soils, Soil and Tillage Research, 103, 98-104.

24. Mouazen, A.M., Kuang, B., De Baerdemaeker, J., Ramon, H., 2010. Comparison between principal component, partial least squares and back propagation neural network analyses for accuracy of measurement of selected soil properties with visible and near infrared spectroscopy. Geoderma, 158, 23-31.

25. Quraishi, M.Z., Mouazen, A.M., 2013a. Calibration of an on-line sensor for measurement of topsoil bulk density in all soil textures. Soil Tillage Research, 126, 219-228.

26. Quraishi, M.Z., Mouazen, A.M., 2013b. Development of a methodology for in situ assessment of topsoil dry bulk density. Soil and Tillage Research, 126, 229-237. 
27. Quraishi, M.Z., Mouazen, A.M., 2013c. Bulk density prediction based on fusion of penetration resistance data with data on soil properties measured with a visible and near-infrared spectrophotometer. British Society of Soil Science, Soil Use and Management Journal, article in review.

28. Slaughter, D.C., Pelletier, M.G., Upadhyaya, S.K., 2001. Sensing soil moisture using NIR spectroscopy, Applied Engineering in Agriculture, 17, 241-247.

29. StatSoft, 2011. STATISTICA (data analysis software system), version 11, StatSoft, Inc., Tulsa, USA.

30. Stenberg, B., Viscarra Rossel, R.A., Mouazen, A.M., Wetterlind, J., 2010. Visible and near infrared spectroscopy in soil science. Advances in Agronomy, 107, 163-215.

31. Stenberg, B., Jensen, L.S., Nordkvist, E., Breland, T.A., Pedersen, A., Guomundsson, J., Bruun, S., Salo, T., Palmason, F., Henriksen, T.M., Korsaeth, A., 2004. Near infrared reflectance spectroscopy for quantification of crop residue, green manure and catch crop $\mathrm{C}$ and $\mathrm{N}$ fractions governing decomposition dynamics in soil, Journal of Near Infrared Spectroscopy, 12, 331-346.

32. Upadhyaya, S.K., Shafii, S., Slaughter, D., 1994. Sensing soil nitrogen for sitespecific crop management (SSCM), ASAE, 94-1055.

33. Viscarra Rossel, R.A., McGlynn, R.N. McBratney, A.B., 2006. Determining the composition of mineral-organic mixes using UV-vis-NIR diffuse reflectance spectroscopy, Geoderma, 137, 70-82.

34. Waiser, T.H., Morgan, C.L.S., 2007. In situ characterization of soil clay content with visible near-infrared diffuse reflectance spectroscopy, Soil Science Society of America Journal, 71, 389-396. 